\title{
Level crossings induced by a longitudinal coupling in the transverse field Ising chain
}

\author{
Grégoire Vionnet, ${ }^{1}$ Brijesh Kumar, ${ }^{2}$ and Frédéric Mila ${ }^{1}$ \\ ${ }^{1}$ Institute of Physics, École Polytechnique Fédérale de Lausanne, CH-1015 Lausanne, Switzerland \\ ${ }^{2}$ School of Physical Sciences, Jawaharlal Nehru University, New Delhi 110067, India
}

(Received 28 January 2017; published 2 May 2017)

\begin{abstract}
We study the effect of antiferromagnetic longitudinal coupling on the one-dimensional transverse field Ising model with nearest-neighbor couplings. In the topological phase where, in the thermodynamic limit, the ground state is twofold degenerate, we show that, for a finite system of $N$ sites, the longitudinal coupling induces $N$ level crossings between the two lowest-lying states as a function of the field. We also provide strong arguments suggesting that these $N$ level crossings all appear simultaneously as soon as the longitudinal coupling is switched on. This conclusion is based on perturbation theory and a mapping of the problem onto the open Kitaev chain, for which we write down the complete solution in terms of Majorana fermions.
\end{abstract}

DOI: 10.1103/PhysRevB.95.174404

\section{INTRODUCTION}

The topological properties of matter are currently attracting considerable attention [1,2]. One of the hallmarks of a topologically nontrivial phase is the presence of surface states. In one dimension, the first example was the spin-1 chain that was shown a long time ago to have a gapped phase [3] with two quasidegenerate low-lying states (a singlet and a triplet) on open chains [4]. These low-lying states are due to the emergent spin-1/2 degrees of freedom at the edges of the chains which combine to make a singlet ground state with an almost degenerate low-lying triplet for an even number of sites and a triplet ground state with an almost degenerate low-lying singlet when the number of sites is odd. In that system, the emergent degrees of freedom are magnetic since they carry a spin $1 / 2$, and they can be detected by standard probes sensitive to local magnetization such as NMR [5].

In fermionic systems, a topological phase is present if the model includes a pairing term (as in the mean-field treatment of a $p$-wave superconductor), and the emergent degrees of freedom are two Majorana fermions localized at the opposite edges of the chain [6]. Their detection is much less easy than that of magnetic edge states, and it relies on indirect consequences such as their impact on the local tunneling density of states $[7,8]$ or the presence of two quasidegenerate low-lying states in open systems. In that respect, looking for situations where the low-lying states cross as a function of an external parameter, for instance, the chemical potential, to prove that there are indeed two low-lying states has been suggested [9].

In a recent experiment with chains of cobalt atoms evaporated onto a $\mathrm{Cu}_{2} \mathrm{~N} / \mathrm{Cu}(100)$ substrate [10], the presence of level crossings as a function of the external magnetic field has been revealed by scanning tunneling microscopy, which exhibits a specific signature whenever the ground state is degenerate. The relevant effective model for that system is a spin-1/2 XY model in an in-plane magnetic field. The exact diagonalization of finite XY chains has indeed revealed the presence of quasidegeneracy between the two lowest-energy states, which are well separated from the rest of the spectrum, and a series of level crossings between them as a function of the magnetic field [11]. Furthermore, the position of these level crossings is in good agreement with the experimental data. It has been proposed that these level crossings are analogous to those predicted in topological fermionic spin chains and that they can be interpreted as a consequence of the Majorana edge modes [12].

The topological phase of the XY model in an in-plane magnetic field is adiabatically connected to that of the transverse field Ising model, in which the longitudinal spin-spin coupling (along the field) is switched off. However, in the transverse field Ising model, the two low-lying states never cross as a function of the field, as can be seen from the magnetization curve calculated by Pfeuty a long time ago [13], which does not show any anomaly. The very different behavior of the XY model in an in-plane field in that respect calls for an explanation. The goal of the present paper is to provide such an explanation and to show that the presence of $N$ level crossings on a chain of $N$ sites is generic as soon as an antiferromagnetic longitudinal coupling is switched on. To achieve this goal, we have studied a Hamiltonian which interpolates between the exactly solvable transverse field Ising (TFI) and the longitudinal field Ising (LFI) chains. The approach that best accounts for these level crossings turns out to be an approximate mapping onto the exactly solvable Kitaev chain, which contains all the relevant physics. In the Majorana representation, the level crossings are due to the interaction between Majorana fermions localized at each end of the chain.

This paper is organized as follows. In Sec. II, we present the model and give some exact diagonalization results on small chains to get an intuition of the qualitative behavior of the spectrum. We show in Sec. III that perturbation theory works in principle but is rather limited because of the difficulty of going to high order. We then turn to an approximate mapping onto the open Kitaev chain via a mean-field decoupling in Sec. IV. The main result of this paper is presented in Sec. V, namely, the explanation of the level crossings in a Majorana representation. Finally, we conclude with a quick discussion of some possible experimental realizations in Sec. VI.

\section{MODEL}

We consider the transverse field spin-1/2 Ising model with an additional antiferromagnetic longitudinal spin-spin 
coupling along the field, i.e., the Hamiltonian

$$
H=J_{x} \sum_{i=1}^{N-1} S_{i}^{x} S_{i+1}^{x}+J_{z} \sum_{i=1}^{N-1} S_{i}^{z} S_{i+1}^{z}-h \sum_{i=1}^{N} S_{i}^{z},
$$

with $J_{z} \geqslant 0$ [14]. This model can be seen as an interpolation between the TFI model $\left(J_{z}=0\right)$ and the LFI model $\left(J_{x}=0\right)$. The case $J_{z}=J_{x}$ corresponds to the effective model describing the experiment in Ref. [10], up to small irrelevant terms [15]. Since we will be mostly interested in the parameter range $0 \leqslant J_{z} \leqslant J_{x}$, we will measure energies in units of $J_{x}$ by setting $J_{x}=1$ henceforth. The spectrum of the Hamiltonian in Eq. (1) is invariant under $h \rightarrow-h$ since the Hamiltonian is invariant if we simultaneously rotate the spins around the $x$ axis so that $S_{i}^{z} \rightarrow-S_{i}^{z} \forall i$. Hence, we will in most cases quote the results only for $h \geqslant 0$.

The TFI limit of $H$ can be solved exactly by Jordan-Wigner mapping onto a chain of spinless fermions [13]. In the thermodynamic limit, it is gapped with a twofold-degenerate ground state for $h<h_{c}=1 / 2$ and undergoes a quantum phase transition at $h=h_{c}$ to a nondegenerate gapped ground state for $h>h_{c}$. The twofold degeneracy when $h<h_{c}$ can be described by two zero-energy Majorana edge modes [6]. As a small positive $J_{z}$ is turned on, there is no qualitative change in the thermodynamic limit, except that $h_{c}$ increases with $J_{z}$. Indeed, the model is then equivalent to the axial next-nearest-neighbor Ising (ANNNI) model in a transverse field which has been extensively studied before (see, for example, $[16,17])$. A second-order perturbation calculation in $1 / h$ yields $h_{c}=1 / 2+(3 / 4) J_{z}+O\left(J_{z}^{2}\right)$ for small $J_{z}$ and $h_{c}=1 / 2+J_{z}+O\left(1 / J_{z}\right)$ for large $J_{z}[18,19]$. Since, for $J_{z} \gtrsim 1$, there are other phases arising [19], we shall mostly consider $J_{z} \lesssim 1$ in the following in order to stay in the phase with a degenerate ground state.

For a finite-size chain, the twofold degeneracy of the TFI model at $0<h<h_{c}$ is lifted, and there is a small nonvanishing energy splitting $\epsilon=E_{1}-E_{0}$ between the two lowest-energy states, where $E_{k}$ are the eigenenergies and $E_{k} \leqslant E_{k+1} \forall k$. This splitting is exponentially suppressed with the system length, $\epsilon \sim \exp (-N / \xi)[6]$. These two quasidegenerate states form a low-energy sector separated from the higher-energy states. The spectrum for $J_{z}=0$ and $N=3$ is shown in Fig. 1(a). For $J_{z}>0$, the splitting $\epsilon$ has an oscillatory behavior and vanishes for some values of $h$. For $N=3$, it vanishes once for $h>0$. See the spectrum for $J_{z}=0.5$ and $J_{z}=1$ in Figs. 1(b) and $1(\mathrm{c})$. As $J_{z}$ becomes large, there is no low-energy sector separated from higher-energy states any more. In the LFI limit, $J_{z} \rightarrow \infty$, the eigenstates have a well-defined magnetization in the $z$ direction, and the energies are linear as a function of $h$ [see Fig. 1(d)]. In this limit, the level crossings are obvious. As the field is increased, the more polarized states become favored, which leads to level crossings.

The plots in Fig. 1 are instructive for very small $N$ but become messy for larger chains. In Figs. 2(a) and 2(b), we show the spectrum relative to the ground-state energy, i.e., $E_{k}-E_{0}$, of a chain of $N=6$ sites for $J_{z}=0$ and $J_{z}=0.75$. The energies $E_{0}$ and $E_{1}$ are plotted in Figs. 2(c) and 2(d) for the same parameters. The structure of the spectrum is similar to the $N=3$ case, except that now $\epsilon$ vanishes at three points for $h>0$. In general, there are $N$ points of exact degeneracy
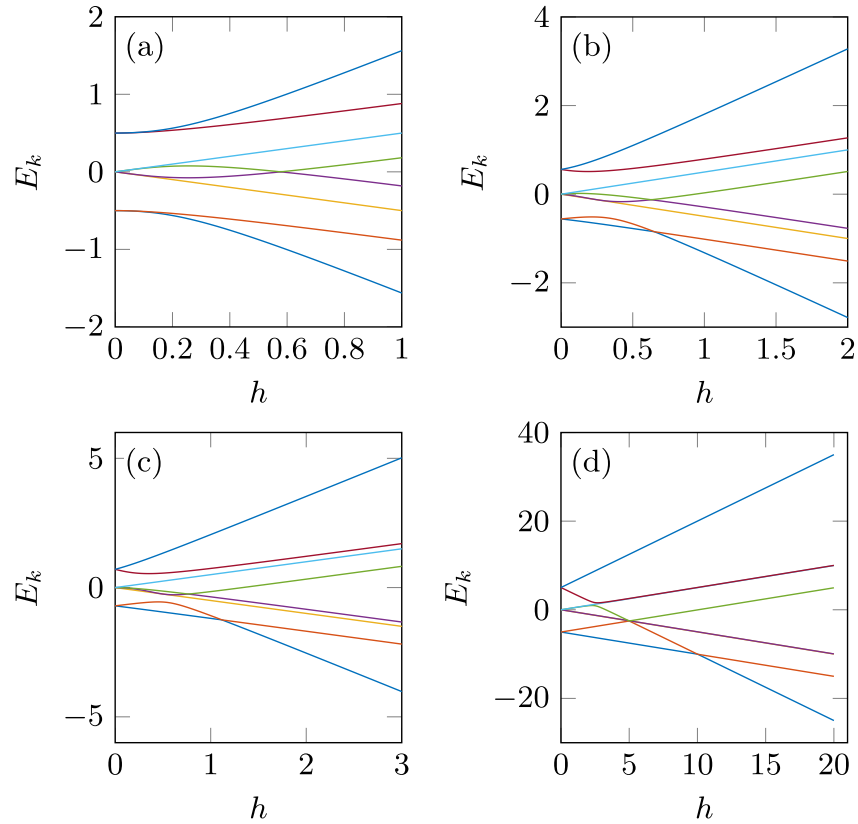

FIG. 1. Exact diagonalization spectrum as a function of $h$ for $N=3$ with (a) $J_{z}=0$, (b) $J_{z}=0.5$, (c) $J_{z}=1$ and (d) $J_{z}=10$.
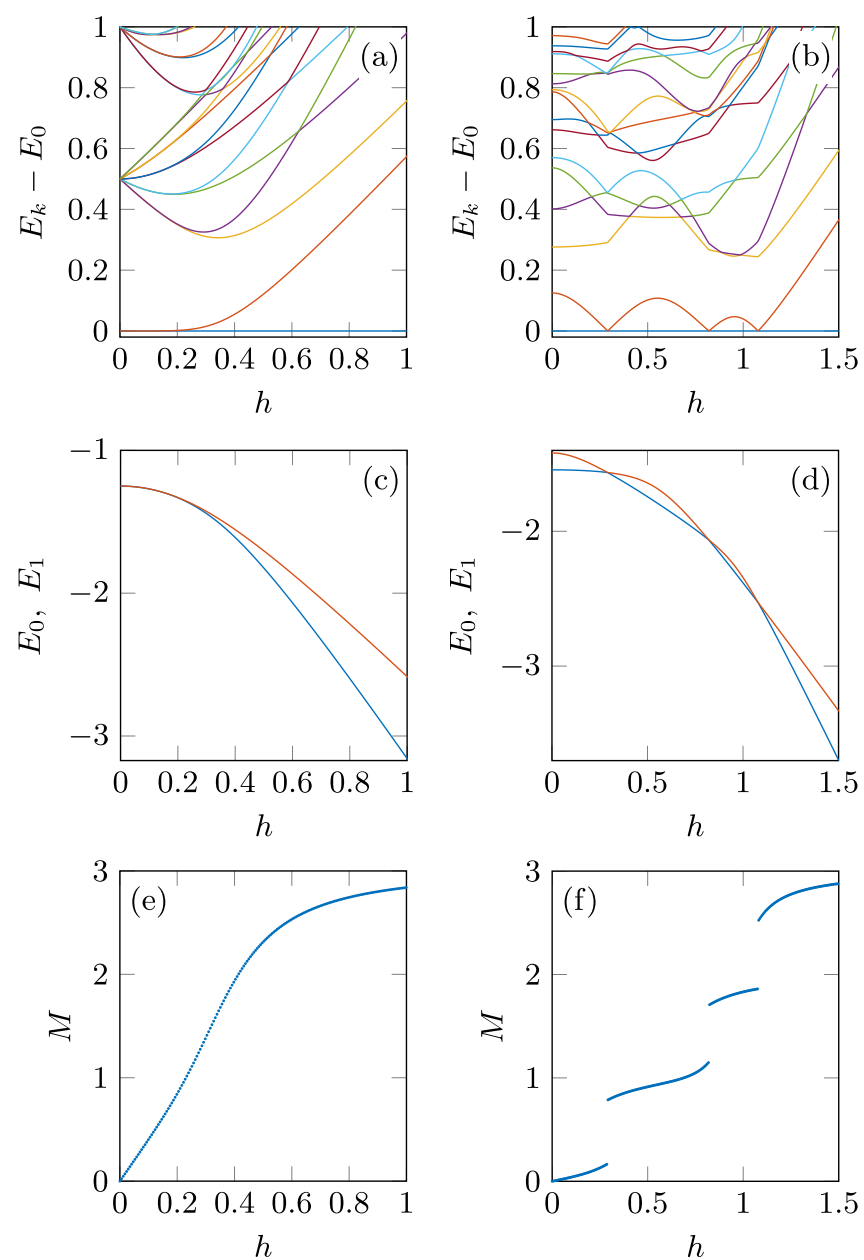

FIG. 2. (a) Exact diagonalization spectrum relative to the groundstate energy, $E_{k}-E_{0}$, (c) the two lowest energies, $E_{0}$ and $E_{1}$, and (e) magnetization $M=-\partial E_{0} / \partial h$ as a function of $h$ for $N=6$ with $J_{z}=0$ (TFI limit). (b), (d), and (f) The same for $J_{z}=0.75$. 


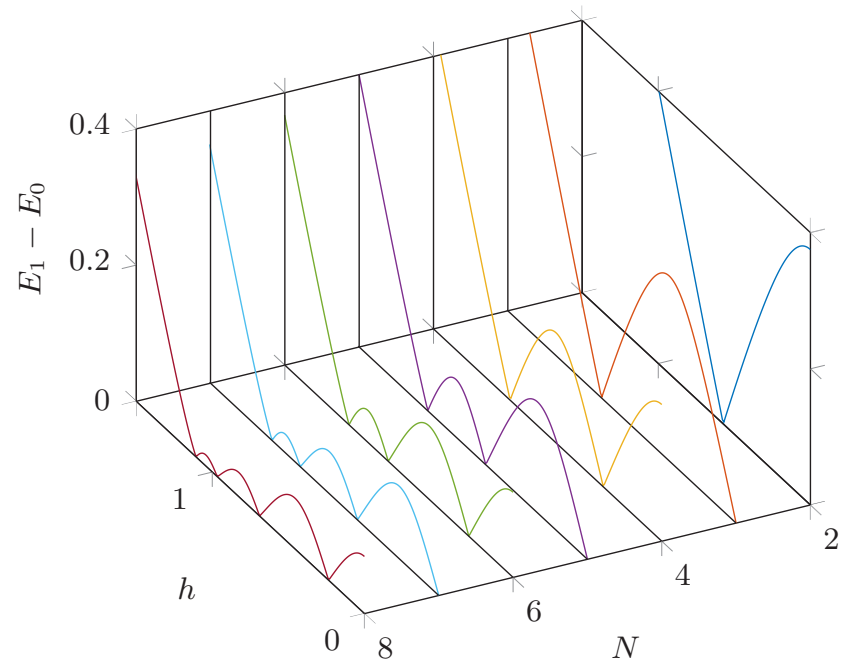

FIG. 3. Exact diagonalization energy splitting $\epsilon=E_{1}-E_{0}$ between the two lowest-energy states as a function of $h$ for several $N$ and $J_{z}=0.75$. There are $N$ level crossings since the spectrum is symmetric under $h \rightarrow-h$.

where the splitting $\epsilon$ vanishes since the spectrum is symmetric under $h \rightarrow-h$. This is shown in Fig. 3 for $2 \leqslant N \leqslant 8$. For even $N$, there are $N / 2$ level crossings for $h>0$, and for odd $N$, there are $(N-1) / 2$ level crossings for $h>0$ and one at $h=0$.

As shown in Figs. 2(e) and 2(f), the level crossings lead to jumps in the magnetization $M(h)=-\partial E_{0} / \partial h$. The number of magnetization jumps turns out to be independent of $J_{z}$ for $0<J_{z}<\infty$, as illustrated in Fig. 4. In the LFI limit, most of the jumps merge together at $h=J_{z}$, with an additional jump persisting for even $N$ at $h=J_{z} / 2$ [20]. In this large- $J_{z}$ region, however, there is no quasidegeneracy, and the magnetization jumps indicate level crossings but no oscillation, in contrast to the small- $J_{z}$ region. Since there are no level crossings in the TFI limit, one might expect the number of crossings to decrease

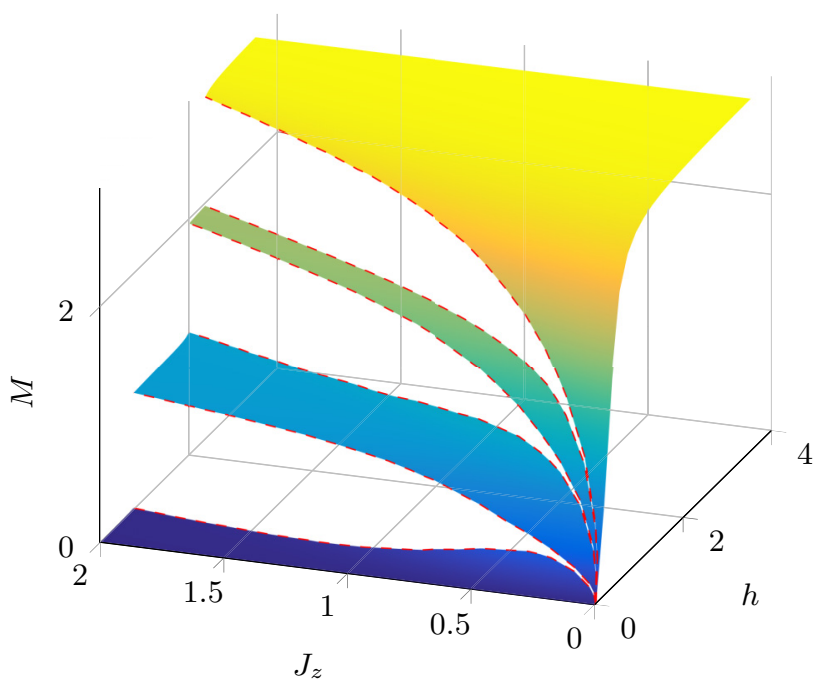

FIG. 4. Exact diagonalization magnetization $M$ as a function of $h$ and $J_{z}$ for $N=6$. The red dashed lines indicate the discontinuities. as $J_{z}$ decreases. However, the exact diagonalization results do not support this scenario and hint at all level crossings appearing at the same time as soon as $J_{z} \neq 0$. This is a remarkable feature that we shall explain in the following.

A useful equivalent representation of the Hamiltonian in Eq. (1) in terms of spinless fermions is obtained by applying the Jordan-Wigner transformation used to solve exactly the TFI model [13],

$$
\begin{aligned}
& S_{i}^{x}=\frac{1}{2}\left(c_{i}^{\dagger}+c_{i}\right) \exp \left(\mathrm{i} \pi \sum_{j<i} c_{j}^{\dagger} c_{j}\right), \\
& S_{i}^{y}=\frac{1}{2 \mathrm{i}}\left(c_{i}^{\dagger}-c_{i}\right) \exp \left(\mathrm{i} \pi \sum_{j<i} c_{j}^{\dagger} c_{j}\right), \\
& S_{i}^{z}=c_{i}^{\dagger} c_{i}-\frac{1}{2},
\end{aligned}
$$

which yields

$$
\begin{aligned}
H= & \frac{1}{4} \sum_{i=1}^{N-1}\left(c_{i}^{\dagger}-c_{i}\right)\left(c_{i+1}^{\dagger}+c_{i+1}\right)-h \sum_{i=1}^{N}\left(c_{i}^{\dagger} c_{i}-\frac{1}{2}\right) \\
& +J_{z} \sum_{i=1}^{N-1}\left(c_{i}^{\dagger} c_{i}-\frac{1}{2}\right)\left(c_{i+1}^{\dagger} c_{i+1}-\frac{1}{2}\right),
\end{aligned}
$$

where $c_{i}$ and $c_{i}^{\dagger}$ are fermionic annihilation and creation operators, respectively. This is the Hamiltonian of a spinless $p$-wave superconductor with nearest-neighbor density-density interaction. As for the simpler TFI model, the Hamiltonian is symmetric under a $\pi$ rotation of the spins around the $z$ axis, $S_{i}^{x} \rightarrow-S_{i}^{x}$ and $S_{i}^{y} \rightarrow-S_{i}^{y}$ in the spin language. This leads to two parity sectors given by the parity operator

$$
P=e^{i \pi \sum_{j=1}^{N} c_{j}^{\dagger} c_{j}}=(-2)^{N} S_{1}^{z} \cdots S_{N}^{z} .
$$

In other words, the Hamiltonian does not mix states with even and odd numbers of up spins, or, equivalently, with even and odd numbers of fermions. The ground-state parity changes at each point of exact degeneracy and thus alternates as a function of the magnetic field for $J_{z}>0$. This can be understood qualitatively by looking at Fig. 2(f). The magnetization plateaus are roughly at $M=0,1,2,3$. Hence, to jump from one plateau to the next, one spin has to flip, thus changing the sign of the parity $P$.

\section{PERTURBATION THEORY}

As a first attempt to understand if the $N$ level crossings develop immediately upon switching on $J_{z}$, we treat the $V=J_{z} \sum_{i=1}^{N-1} S_{i}^{z} S_{i+1}^{z}$ term as a perturbation to the exactly solvable transverse field Ising model. One may naively expect that degenerate perturbation theory is required since the TFI chain has a quasi-twofold degeneracy at low field. Fortunately, the two low-energy states live in different parity sectors [13] that are not mixed by the perturbation $V$. We can therefore apply the simple Rayleigh-Schrödinger perturbation theory in the range of parameters we are interested in, i.e., $J_{z} \lesssim 1$.

Writing $A_{i}=c_{i}^{\dagger}+c_{i}$ and $B_{i}=c_{i}^{\dagger}-c_{i}$, the perturbation can be rewritten as $V=\left(J_{z} / 4\right) \sum_{i=1}^{N-1} B_{i} A_{i} B_{i+1} A_{i+1}$. The 

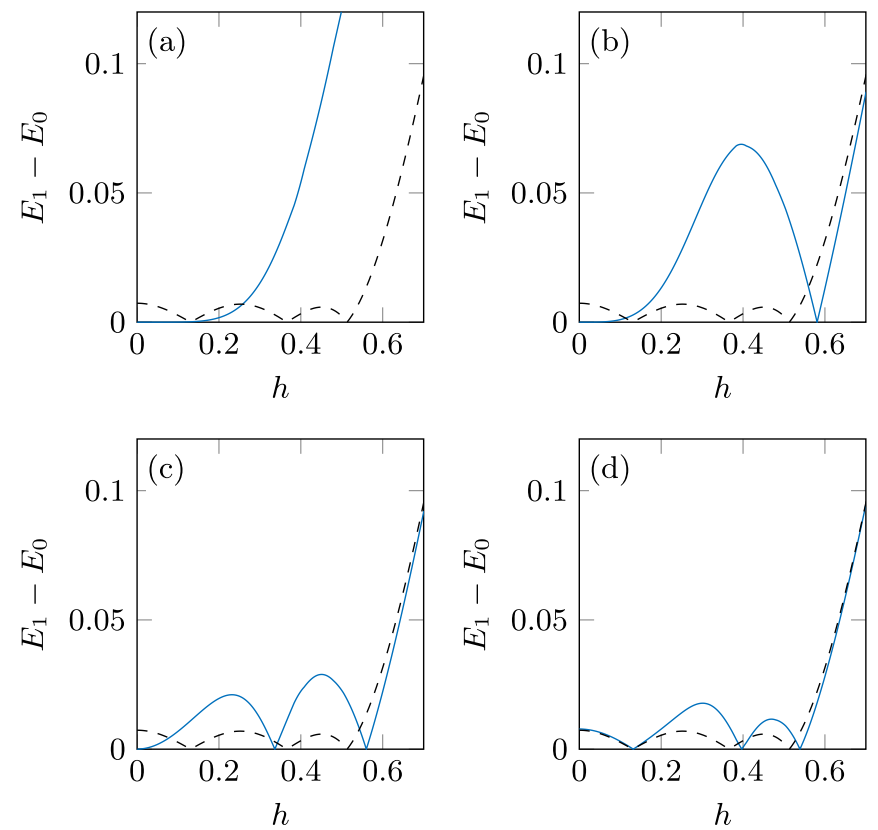

FIG. 5. Energy splitting at (a) zeroth, (b) first, (c) second, and (d) third order (blue solid lines) compared to the exact diagonalization result (black dashed lines) for $N=6$ and $J_{z}=0.25$.

unperturbed eigenstates are $|m\rangle=\Upsilon_{m}^{\dagger}|0\rangle$, where $|0\rangle$ is the ground state and $\Upsilon_{m}^{\dagger}$ are a product of the creation operators corresponding to the Bogoliubov fermions. The matrix elements are then

$$
\langle n|V| m\rangle=\frac{J_{z}}{4} \sum_{i=1}^{N-1}\left\langle 0\left|\Upsilon_{n} B_{i} A_{i} B_{i+1} A_{i+1} \Upsilon_{m}^{\dagger}\right| 0\right\rangle,
$$

which can be computed by applying Wick's theorem in a way similar to how correlation functions are found in [21]. We computed the effect of $V$ up to third order with the basis of virtual states slightly truncated, namely, by keeping states with at most three Bogoliubov fermions. Since the more fermions there are in a state, the larger its energy is, we expect this approximation to be excellent.

As shown in Fig. 5, the number of crossings increases with the order of perturbation, and to third order in perturbation, the results for $N=6$ sites are in qualitative agreement with exact diagonalizations. From the way level crossings appear upon increasing the order of perturbation theory, one can expect to induce up to $2 m+1$ level crossings if perturbation theory is pushed to order $m$ (see Fig. 6). So these results suggest that the appearance of level crossings is a perturbative effect and that, for a given size $N$, pushing perturbation theory to high enough order will indeed lead to $N$ level crossings for small $J_{z}$. However, in practice, it is impossible to push perturbation theory to very high order. Indeed, the results at order 3 are already very demanding. So these perturbative results are encouraging, but they call for an alternative approach to actually prove that the number of level crossings is indeed equal to $N$ and that these level crossings appear as soon as $J_{z}$ is switched on.

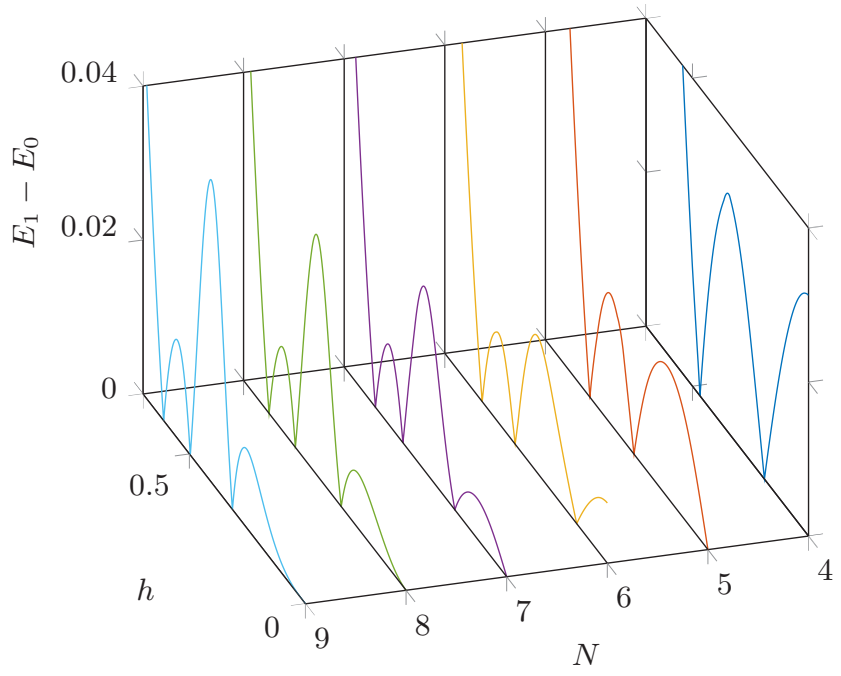

FIG. 6. Energy splitting at third order in perturbation theory for several $N$ and $J_{z}=0.25$. Since the spectrum is symmetric under $h \rightarrow-h$, there are $N$ level crossings for $N \leqslant 7$ and seven level crossings for $N \geqslant 7$.

\section{FERMIONIC MEAN-FIELD APPROXIMATION}

In the fermionic representation, Eq. (3), there is a quartic term that cannot be treated exactly. Here, we approximate it by mean-field decoupling. In such an approximation, one assumes the system can be well approximated by a noninteracting system (quadratic in fermions) with self-consistently determined parameters. For generality, we decouple the quartic term in all three mean-field channels consistent with Wick's theorem,

$$
\begin{aligned}
c_{i}^{\dagger} c_{i} c_{i+1}^{\dagger} c_{i+1} & \\
\approx & \left\langle c_{i}^{\dagger} c_{i}\right\rangle c_{i+1}^{\dagger} c_{i+1}+\left\langle c_{i+1}^{\dagger} c_{i+1}\right\rangle c_{i}^{\dagger} c_{i}-\left\langle c_{i}^{\dagger} c_{i}\right\rangle\left\langle c_{i+1}^{\dagger} c_{i+1}\right\rangle \\
& -\left\langle c_{i}^{\dagger} c_{i+1}^{\dagger}\right\rangle c_{i} c_{i+1}-\left\langle c_{i} c_{i+1}\right\rangle c_{i}^{\dagger} c_{i+1}^{\dagger}+\left\langle c_{i}^{\dagger} c_{i+1}^{\dagger}\right\rangle\left\langle c_{i} c_{i+1}\right\rangle \\
& +\left\langle c_{i}^{\dagger} c_{i+1}\right\rangle c_{i} c_{i+1}^{\dagger}+\left\langle c_{i} c_{i+1}^{\dagger}\right\rangle c_{i}^{\dagger} c_{i+1}-\left\langle c_{i}^{\dagger} c_{i+1}\right\rangle\left\langle c_{i} c_{i+1}^{\dagger}\right\rangle .
\end{aligned}
$$

Here, $\langle\cdot\rangle$ denotes the ground-state expectation value. The $3 N-$ 2 self-consistent parameters $\left\langle c_{i}^{\dagger} c_{i}\right\rangle,\left\langle c_{i}^{\dagger} c_{i+1}^{\dagger}\right\rangle$, and $\left\langle c_{i}^{\dagger} c_{i+1}\right\rangle$ can be found straightforwardly by iteratively solving the quadratic mean-field Hamiltonian.

As it turns out, it is more instructive to consider only three self-consistent parameters. To do so, we solve the mean-field approximation of the translationally invariant Hamiltonian $\left(c_{N+1}=c_{1}\right)$,

$$
\begin{aligned}
H^{\prime}= & \sum_{i=1}^{N}\left\{\frac{1}{4}\left(c_{i}^{\dagger}-c_{i}\right)\left(c_{i+1}^{\dagger}+c_{i+1}\right)-h\left(c_{i}^{\dagger} c_{i}-\frac{1}{2}\right)\right. \\
& \left.+J_{z}\left(c_{i}^{\dagger} c_{i}-\frac{1}{2}\right)\left(c_{i+1}^{\dagger} c_{i+1}-\frac{1}{2}\right)\right\} \\
\approx & \sum_{i=1}^{N}\left\{-\mu c_{i}^{\dagger} c_{i}+\left(t c_{i+1}^{\dagger} c_{i}+\text { H.c. }\right)-\left(\Delta c_{i+1}^{\dagger} c_{i}^{\dagger}+\text { H.c. }\right)\right\} \\
& + \text { const },
\end{aligned}
$$



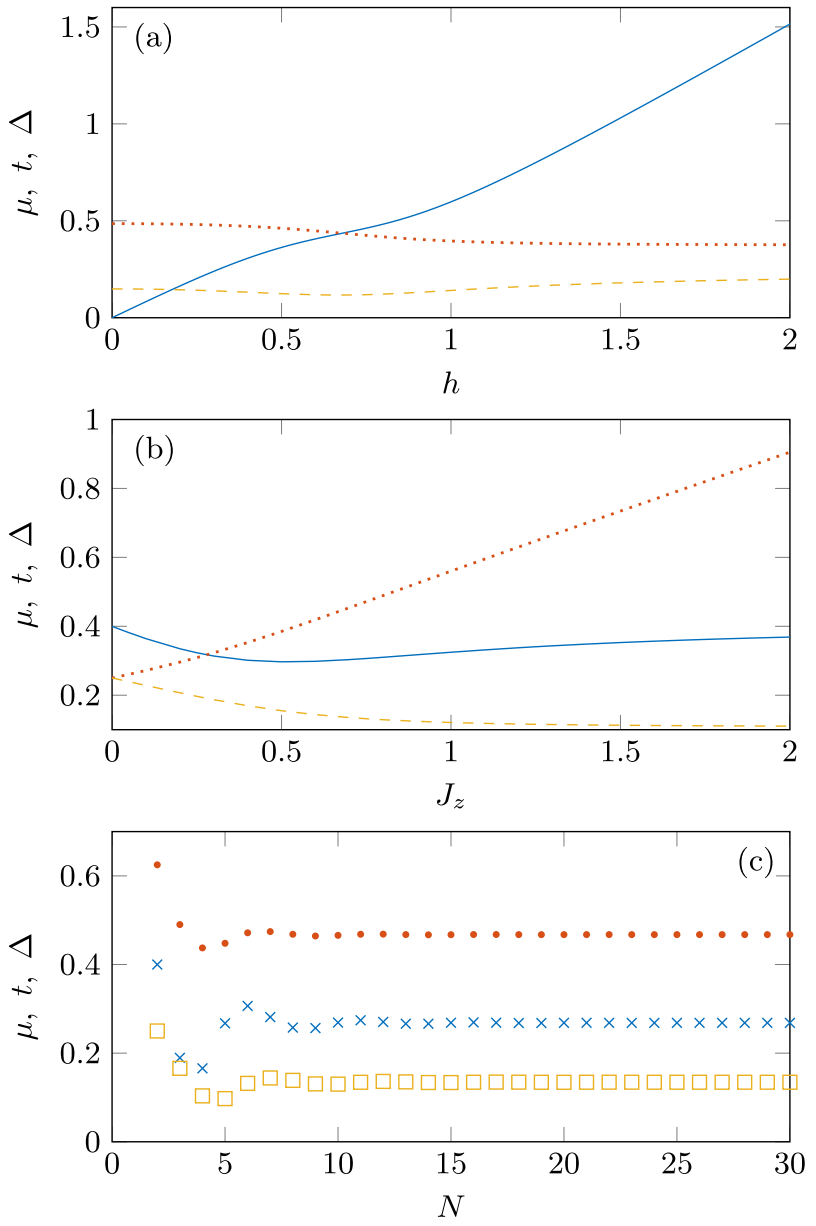

FIG. 7. Self-consistent mean-field parameters $\mu$ (blue solid lines and crosses), $t$ (red dotted lines and dots), and $\Delta$ (yellow dashed lines and squares) (a) as a function of $h$ for $J_{z}=0.75$ and $N=6$, (b) as a function of $J_{z}$ for $h=0.4$ and $N=6$, and (c) as a function of $N$ for $h=0.4$ and $J_{z}=0.75$.

where $\mu=h+J_{z}\left(1-2\left\langle c_{i}^{\dagger} c_{i}\right\rangle\right), t=1 / 4-J_{z}\left\langle c_{i}^{\dagger} c_{i+1}\right\rangle$, and $\Delta=1 / 4-J_{z}\left\langle c_{i} c_{i+1}\right\rangle$ are determined self-consistently. These parameters are found to be real and are shown in Fig. 7 as a function $h, J_{z}$, and $N$.

Using these self-consistent parameters, the Hamiltonian in Eq. (3) is then approximated by the following mean-field problem on an open chain:

$$
\begin{aligned}
H_{\mathrm{MF}}= & -\sum_{i=1}^{N} \mu\left(c_{i}^{\dagger} c_{i}-\frac{1}{2}\right) \\
& +\sum_{i=1}^{N-1}\left[\left(t c_{i+1}^{\dagger} c_{i}+\text { H.c. }\right)-\left(\Delta c_{i+1}^{\dagger} c_{i}^{\dagger}+\text { H.c. }\right)\right],
\end{aligned}
$$

up to an irrelevant additive constant [22].

Since the self-consistent parameters are almost independent of the system size [see Fig. 7(c)], the boundaries are not very important, and the bulk contribution is determinant. This partly justifies the approximation of playing with the boundary conditions to get the approximate model (8) with just three selfconsistent parameters. This approximation is also justified by the great quantitative agreement with the exact diagonalization
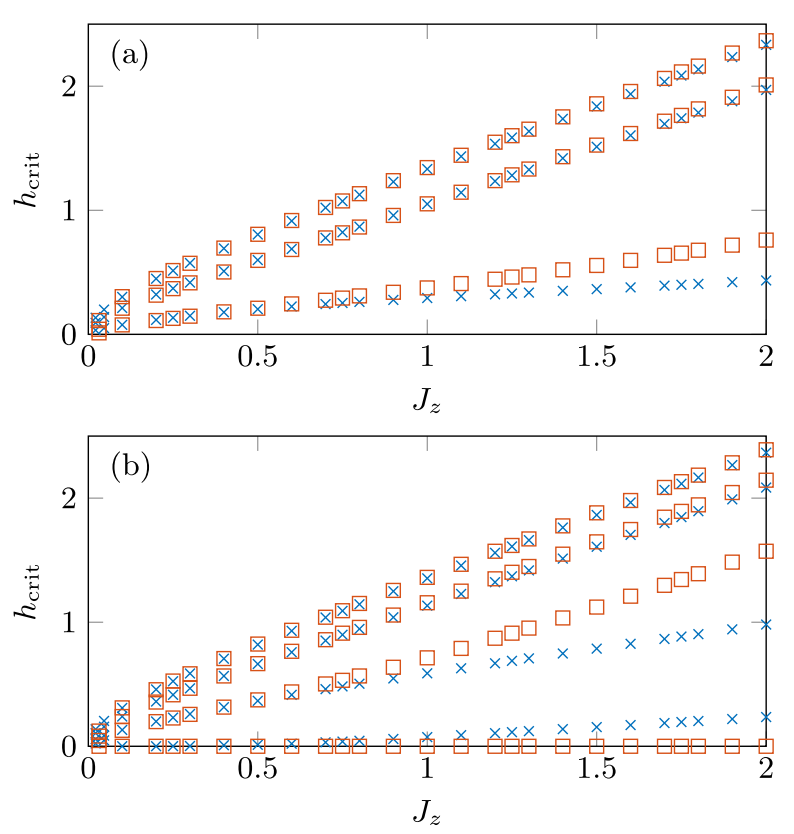

FIG. 8. Critical fields $h_{\text {crit }}$ where the degeneracy is exact as a function of $J_{z}$ in the self-consistent mean-field approximation (8) (blue crosses) compared to the exact diagonalization result (red squares) for (a) $N=6$ and (b) $N=7$.

results for the critical fields for $J_{z} \lesssim 0.8$ (see Fig. 8) and, to a lesser extent, for the energy splitting $\epsilon=E_{1}-E_{0}$ between the two lowest-energy states (see Fig. 9).

For odd $N$, the degeneracy at $h=0$ is protected by symmetry for any $J_{z}$ in the Hamiltonian (1). Indeed, under the transformation $S_{i}^{z} \rightarrow-S_{i}^{z} \forall i$, the parity operator transforms as $P \rightarrow(-1)^{N} P$. Hence, for odd $N$ and $h=0$, the ground state
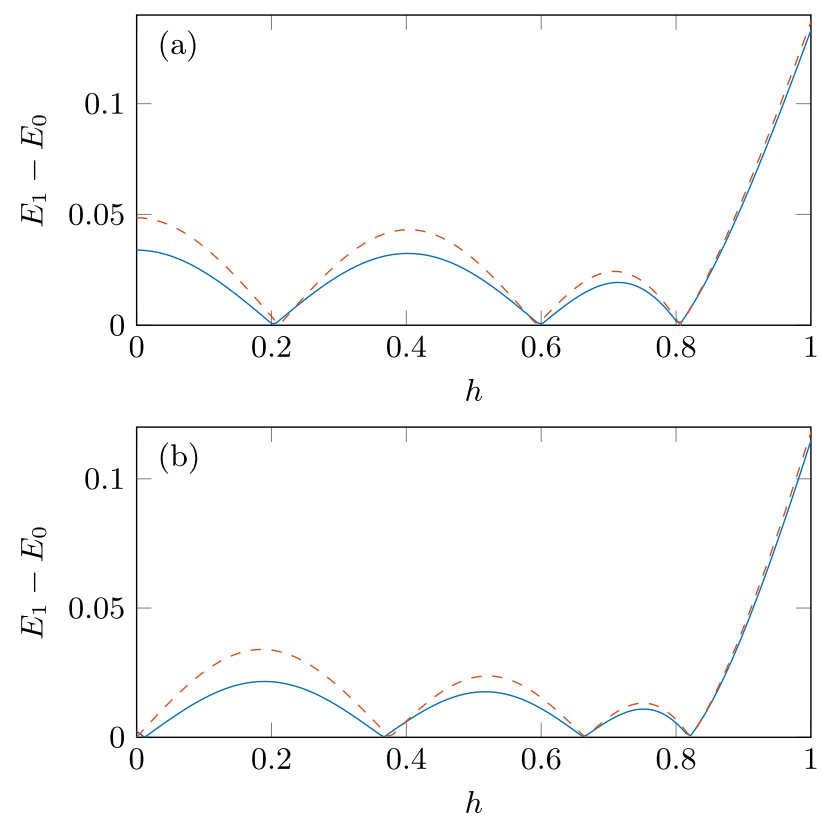

FIG. 9. Energy splitting $\epsilon=E_{1}-E_{0}$ as a function of $h$ in the self-consistent mean-field approximation (8) (blue solid line) compared to the exact diagonalization result (red dashed line) for $J_{z}=0.5$ and (a) $N=6$ and (b) $N=7$. 
has to be twofold degenerate. As can be seen in Fig. 8(b), the critical field $h=0$ at low $J_{z}$ evolves to a nonzero value for large $J_{z}$, thus showing that this symmetry is broken by the mean-field approximation (8). The discrepancy is, however, small for $J_{z} \lesssim 0.8$, as can also be seen in Fig. 9(b).

We observe from Fig. 7(a) that as a function of magnetic field, the parameters $t$ and $\Delta$ are almost constant, whereas $\mu$ is almost proportional to $h$. Thus, we can understand the physics of the level oscillations by forgetting about the self-consistency and considering $\mu, t$, and $\Delta$ as free parameters, i.e., by studying the open Kitaev chain [6], where the level crossings happen as $\mu$ is tuned. Compared to the TFI model for which $\Delta=t$, the main effect of $J_{z}>0$ is to make $0<\Delta<t$, which, as we shall see in the next section, is the condition to see level oscillations.

Such a mapping between the two lowest-lying energy states of the interacting Kitaev chain and of the noninteracting Kitaev chain can be made rigorous for a special value of $h>0$, provided the boundary terms in Eq. (3) are slightly modified [23]. But this particular exact case misses out on level-crossing oscillations.

\section{LEVEL OSCILLATIONS AND MAJORANA FERMIONS}

We define $2 N$ Majorana operators $\gamma_{i}^{\prime}, \gamma_{i}^{\prime \prime}$ as

$$
\begin{array}{r}
\gamma_{i}^{\prime}=c_{i}+c_{i}^{\dagger}, \\
\gamma_{i}^{\prime \prime}=-\mathrm{i}\left(c_{i}-c_{i}^{\dagger}\right),
\end{array}
$$

which satisfy $\gamma_{i}^{\prime \dagger}=\gamma_{i}^{\prime}, \gamma_{i}^{\prime \prime \dagger}=\gamma_{i}^{\prime \prime},\left\{\gamma_{i}^{\prime}, \gamma_{j}^{\prime \prime}\right\}=0$, and $\left\{\gamma_{i}^{\prime}, \gamma_{j}^{\prime}\right\}=$ $\left\{\gamma_{i}^{\prime \prime}, \gamma_{j}^{\prime \prime}\right\}=2 \delta_{i j}$. Since $\mu, t$, and $\Delta$ are real, $H_{M F}$ of Eq. (8) reads

$$
\begin{aligned}
H_{\mathrm{MF}}= & \frac{i}{2} \sum_{i=1}^{N-1}\left[-(t+\Delta) \gamma_{i}^{\prime \prime} \gamma_{i+1}^{\prime}+(t-\Delta) \gamma_{i}^{\prime} \gamma_{i+1}^{\prime \prime}\right] \\
& -\frac{i \mu}{2} \sum_{i=1}^{N} \gamma_{i}^{\prime} \gamma_{i}^{\prime \prime}=\frac{i}{2} \sum_{i, j=1}^{N} \gamma_{i}^{\prime} M_{i j} \gamma_{j}^{\prime \prime}
\end{aligned}
$$

From the singular-value decomposition of $M$, we write $M=$ $U \Sigma V^{T}$, where $U$ and $V$ are orthogonal matrices and $\Sigma=$ $\operatorname{diag}\left(\epsilon_{1}, \ldots, \epsilon_{N}\right)$, with real $\epsilon_{i}$ and $\left|\epsilon_{i}\right| \leqslant\left|\epsilon_{i+1}\right| \forall i$. Thus, the Hamiltonian reads

$$
\begin{aligned}
H_{\mathrm{MF}} & =\frac{i}{2} \sum_{i, j, k=1}^{N} \gamma_{i}^{\prime} U_{i k} \epsilon_{k} V_{k j}^{T} \gamma_{j}^{\prime \prime}=\frac{i}{2} \sum_{k=1}^{N} \epsilon_{k} \tilde{\gamma}_{k}^{\prime} \tilde{\gamma}_{k}^{\prime \prime} \\
& =\sum_{k} \epsilon_{k}\left(\eta_{k}^{\dagger} \eta_{k}-\frac{1}{2}\right),
\end{aligned}
$$

where

$$
\tilde{\gamma}_{k}^{\prime}=\sum_{i=1}^{N} \gamma_{i}^{\prime} U_{i k}, \quad \tilde{\gamma}_{k}^{\prime \prime}=\sum_{i=1}^{N} \gamma_{i}^{\prime \prime} V_{i k}
$$

are the rotated Majorana operators and $\eta_{k}=\frac{1}{2}\left(\tilde{\gamma}_{k}^{\prime}+\mathrm{i} \tilde{\gamma}_{k}^{\prime \prime}\right)$ are fermionic annihilation operators corresponding to the Bogoliubov quasiparticles.
As derived in the Appendix, in general the Majorana operators, $\tilde{\gamma}_{k}^{\prime}$ and $\tilde{\gamma}_{k}^{\prime \prime}$, are of the form

$$
\begin{aligned}
& \tilde{\gamma}_{k}^{\prime}=\sum_{j}\left(a_{+} x_{+}^{j}+b_{+} x_{+}^{N+1-j}+a_{-} x_{-}^{j}+b_{-} x_{-}^{N+1-j}\right) \gamma_{j}^{\prime}, \\
& \tilde{\gamma}_{k}^{\prime \prime}=\sum_{j}\left(a_{+} x_{+}^{N+1-j}+b_{+} x_{+}^{j}+a_{-} x_{-}^{N+1-j}+b_{-} x_{-}^{j}\right) \gamma_{j}^{\prime \prime},
\end{aligned}
$$

where $x_{ \pm}, a_{ \pm}$, and $b_{ \pm}$are functions of the energy $\epsilon_{k}$, which is quantized in order to satisfy the boundary conditions. On can easily solve numerically the nonlinear equation for $\epsilon_{k}$. Here, we will instead focus on a simple analytical approximation for $\tilde{\gamma}_{1}^{\prime}, \tilde{\gamma}_{1}^{\prime \prime}$, and $\epsilon_{1}$, which works well to discuss the level crossings and is equivalent to the ansatz given in [6].

From Eqs. (A4) and (A5), we see that for $\epsilon=0$, we have either $a_{ \pm}=0$ or $b_{ \pm}=0$. Without loss of generality, we can choose $b_{ \pm}(\epsilon=0)=0$. Since we expect $\epsilon_{1} \ll 1$, we approximate

$$
b_{ \pm}\left(\epsilon_{1}\right) \approx b_{ \pm}(0)=0
$$

and

$$
x_{ \pm}\left(\epsilon_{1}\right) \approx x_{ \pm}(0)=\frac{\mu \pm \sqrt{\mu^{2}-4 t^{2}+4 \Delta^{2}}}{2(t+\Delta)},
$$

which yields

$$
\begin{aligned}
& \tilde{\gamma}_{1}^{\prime} \approx \sum_{j}\left(a_{+} x_{+}^{j}+a_{-} x_{-}^{j}\right) \gamma_{j}^{\prime}, \\
& \tilde{\gamma}_{1}^{\prime \prime} \approx \sum_{j}\left(a_{+} x_{+}^{N+1-j}+a_{-} x_{-}^{N+1-j}\right) \gamma_{j}^{\prime \prime},
\end{aligned}
$$

with $\sum_{j}\left(a_{+} x_{+}^{j}+a_{-} x_{-}^{j}\right)^{2}=1$. The boundary conditions (A9) now read

$$
\begin{aligned}
a_{+}+a_{-} & =0, \\
a_{+} x_{+}^{N+1}+a_{-} x_{-}^{N+1} & =0,
\end{aligned}
$$

and in general both cannot be satisfied unless $\epsilon_{1}=0$ exactly.

If $\left|x_{ \pm}\right|<1, \quad \tilde{\gamma}_{1}^{\prime}$ is localized on the left side of the chain with its amplitude $\sim e^{-j / \xi}$ as $j \gg 1$, with $\xi=$ $-1 / \ln \left[\max \left(\left|x_{+}\right|,\left|x_{-}\right|\right)\right]$. Furthermore, $\tilde{\gamma}_{1}^{\prime \prime}$ is related to $\tilde{\gamma}_{1}^{\prime}$ by the reflection symmetry $j \rightarrow N+1-j$. Thus, in the thermodynamic limit, the boundary condition (17b) is irrelevant, and $\epsilon_{1} \rightarrow 0$ as $N \rightarrow \infty$. Similarly, if $\left|x_{ \pm}\right|>1$, the boundary condition (17a) becomes irrelevant in the thermodynamic limit. However, if $\left|x_{+}\right|>1$ and $\left|x_{-}\right|<1$ or $\left|x_{+}\right|<1$ and $\left|x_{-}\right|>1$, then $\tilde{\gamma}_{1}^{\prime}$ and $\tilde{\gamma}_{1}^{\prime \prime}$ have significant weight on both sides of the chain, and both boundary conditions (17a) and (17b) remain important in the thermodynamic limit. Hence, the approximation $\epsilon_{1} \approx 0$ is bad, indicating a gapped system. As discussed in [6], for $|\mu|<2|t|$ we have either $\left|x_{ \pm}\right|<1$ or $\left|x_{ \pm}\right|>1$, which yields $\epsilon_{1}=0$ in the thermodynamic limit. This is the topological phase with a twofold-degenerate ground state. For a finite system, however, the boundary conditions (17a) and (17b) are, in general, not exactly satisfied, and the system is only quasidegenerate with a gap $\epsilon \sim e^{-N / \xi}$. For $|\mu|>2|t|$, either $\left|x_{+}\right|>1$ and $\left|x_{-}\right|<1$ or $\left|x_{+}\right|<1$ and $\left|x_{-}\right|>1$, and the system is gapped. 

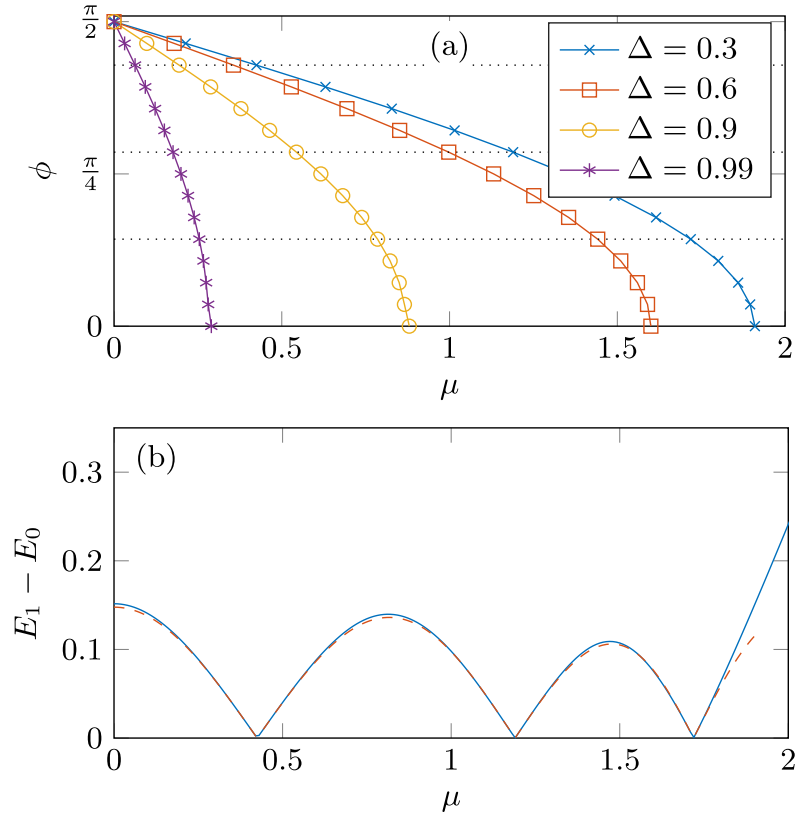

FIG. 10. (a) Phase $\phi(\mu)$ of $x_{+}=r e^{\mathrm{i} \phi}$ within the approximation (15) for several $\Delta$ with $t=1$ and $N=6$. The horizontal black dotted lines indicate the values $\phi=\frac{\pi m}{N+1}$. (b) Splitting $E_{1}-E_{0}=\left|\epsilon_{1}\right|$ in the Kitaev chain calculated exactly solving numerically the full selfconsistent equations described in the Appendix (blue solid line) and with the analytical approximate result in Eq. (19) (red dashed line) for $N=6, t=1$, and $\Delta=0.3$.

In the topological phase, $|\mu|<2|t|$, there are parameters for which the boundary conditions (17) can be exactly satisfied even for $N<\infty$ and thus $\epsilon_{1}=0$ exactly. In such a case, there is an exact zero mode even for a finite chain. This was previously discussed in Ref. [24], as well as in [25], where a more general method that applies to disordered systems is described. If $x_{ \pm} \in \mathbb{R}$, it is never possible to satisfy the boundary conditions (17), and therefore, the quasigap is always finite, $\epsilon_{1} \neq 0$. However, if $x_{+}=r e^{i \phi} \notin \mathbb{R}$, Eq. (15) yields $x_{-}=x_{+}^{*}$ and $\left(x_{+}^{N+1}-x_{-}^{N+1}\right) \propto r^{N+1} \sin [(N+1) \phi]$. Thus, it may happen for specific parameters that $\epsilon_{1}=0$ exactly. This degeneracy indicates a level crossing. The phase $\phi$, defined for $|\mu|<\mu_{c}=2 \sqrt{t^{2}-\Delta^{2}}$, is given by

$$
\tan \phi=\sqrt{\left(\mu_{c} / \mu\right)^{2}-1} .
$$

It thus goes continuously from $\phi\left(\mu=0^{+}\right)=\pi / 2$ to $\phi(\mu \rightarrow$ $\left.\mu_{c}\right) \rightarrow 0$. Hence, there are critical chemical potentials, $0 \leqslant \mu_{\lceil N / 2\rceil}<\cdots<\mu_{m}<\cdots<\mu_{1}<\mu_{c}$, such that $\phi(\mu=$ $\left.\mu_{m}\right)=\frac{\pi m}{N+1}$ [see Fig. 10(a)]. For these critical $\mu_{m}$, the system is exactly degenerate, i.e., $\epsilon_{1}=0$. In the TFI limit, we have $\Delta=t$ and $\mu_{c}=0$; thus, there are no level crossings.

For $|\mu|<2|t|$, writing $x_{+}=r e^{i \phi}$, with $r>0$, we have

$$
\begin{aligned}
\epsilon_{1} & =\Sigma_{11}=\left(U^{T} M V\right)_{11} \\
& \approx 4(t+\Delta) a_{+}^{2} r^{N+2} \sin (\phi) \sin [(N+1) \phi],
\end{aligned}
$$

where we used the approximations (15) and (16) and the boundary condition (17a) [(17b)] when $t \Delta>0(t \Delta<0)$ since in this case $\left|x_{ \pm}\right|<1\left(\left|x_{ \pm}\right|>1\right)$. Note that $\phi(-\mu)=$ $\phi(\mu)-\pi$, and thus, $\epsilon_{1}$ is an odd function of $\mu$ for odd $N$ and an even function of $\mu$ for even $N$. Since $\epsilon_{1}$ changes sign whenever $\sin [(N+1) \phi]=0$, the degeneracy points indicate level crossings. This approximate description works extremely well, as shown in Fig. 10(b) for $\Delta=0.3 t$. Because $\phi$ takes all the values in $] 0, \pi / 2$ ] for $0<\mu<\mu_{c}$ and in ] $-\pi,-\pi / 2$ ] for $-\mu_{c}<\mu<0$, there are either exactly $N$ level crossings as a function of $\mu$ if $0<\mu_{c} \in \mathbb{R}$, i.e., if $|\Delta|<|t|$, and no zero level crossings otherwise. At the points of exact degeneracy, $b_{ \pm}(\epsilon=$ $0)=0$, the zero-mode Majorana fermions are localized on opposite sides of the chain. When the degeneracy is not exact, however, $b_{ \pm}(\epsilon \neq 0) \neq 0$, and the zero-mode Majorana fermions mix together to form Majoranas localized mostly on one side but also a little bit on the opposite side.

In the XY model in an out-of-plane magnetic field, which is equivalent to the noninteracting Kitaev chain [21], these level crossings lead to an oscillatory behavior of the spin correlation functions [26]. In the context of $p$-wave superconductors, the level oscillations described above also arise in more realistic models and are considered one of the hallmarks of the presence of topological Majorana fermions [9,27]. Although it is still being debated whether Majorana fermions have already been observed, strong experimental evidence for the level oscillations was reported in [28].

Coming back to the mean-field Hamiltonian of Eq. (6), we can get the phase $\phi$ within the approximation (15), i.e., the phase of $x_{+}(\epsilon=0)$, as a function of the physical parameters $h$ and $J_{z}$ since we know how the self-consistent parameters $\mu, t$, and $\Delta$ depend on them. We plot in Fig. 11 the phase $\phi$ as a function of $h$ for several $J_{z}$, which yields a good qualitative understanding of the sudden appearance of $N$ level crossings as soon as $J_{z}>0$. As previously discussed, the self-consistent parameters are almost independent of $N$, and therefore, the curves $\phi(h)$ are almost independent of $N$ as well. The main effect of $N$ is to change the condition $\phi\left(\mu=\mu_{m}\right)=\frac{\pi m}{N+1}$ for the boundary condition in Eq. (17b) to be satisfied and thus for the system to be exactly degenerate.

\section{SUMMARY}

The main result of this paper is that the level crossings between the two lowest-energy eigenstates of the XY chain in an in-plane magnetic field are more generally a fundamental feature of the transverse field Ising chain with an antiferromagnetic longitudinal coupling however small. These points of level crossings (twofold degeneracy) correspond to having Majorana edge modes in a Kitaev chain onto which the problem can be approximately mapped. The level crossings of the XY chains have been observed experimentally in [10] by scanning tunneling microscopy on cobalt atoms evaporated onto a $\mathrm{Cu}_{2} \mathrm{~N} / \mathrm{Cu}(100)$ substrate. By varying the adsorbed atoms and the substrate, it should be possible to vary the easy-plane and easy-axis anisotropies and thus to explore the exact degeneracy points for various values of the longitudinal coupling. The possibility to probe the twofold degeneracy of this family of spin chains is important in view of their potential use for universal quantum computation [29]. Further, one could also realize the spinless fermionic Hamiltonian (3) in an array of Josephson junctions as described in [19]. The advantage of this realization is that it allows great flexibility to tune all the parameters of the model. We hope that the results of the 

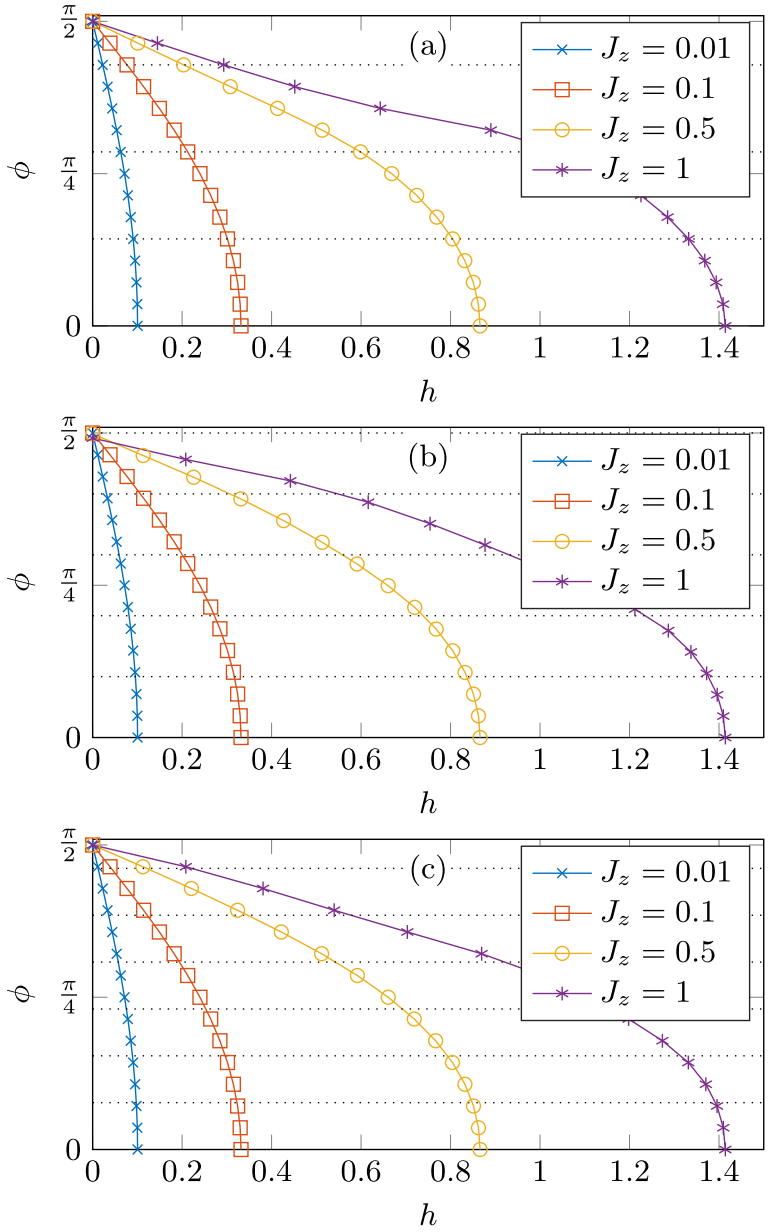

FIG. 11. Phase $\phi(h)$ of $x_{+}(\epsilon=0)$ based on the self-consistent parameters $\mu, t, \Delta$ of the mean-field decoupling for several $J_{z}$ and (a) $N=6$, (b) $N=9$, and (c) $N=12$. The horizontal black dotted lines indicate the values $\phi=\frac{\pi m}{N+1}$.

present paper will stimulate experimental investigations along these lines.

\section{ACKNOWLEDGMENTS}

We acknowledge S. Jalal for useful discussions and the Swiss National Science Foundation for financial support. B.K. acknowledges the financial support from the UPE-II and DSTPURSE programs of JNU.

\section{APPENDIX: MAJORANA SOLUTIONS OF THE KITAEV CHAIN}

To solve the Kitaev chain (8), we need to find the singularvalue decomposition of

$$
M=\left(\begin{array}{cccccc}
-\mu & \tau_{-} & 0 & \ldots & & \\
\tau_{+} & -\mu & \tau_{-} & 0 & \ldots & \\
0 & \tau_{+} & -\mu & \tau_{-} & 0 & \ldots \\
& & \ddots & \ddots & \ddots & \\
& \ldots & 0 & \tau_{+} & -\mu & \tau_{-} \\
& & \ldots & 0 & \tau_{+} & -\mu
\end{array}\right),
$$

with $\tau_{ \pm}=t \pm \Delta$, i.e., find orthogonal matrices $U$ and $V$ and a real diagonal matrix $\Sigma$ such that $M=U \Sigma V^{T}$. Writing $\vec{u}_{k}$ and $\vec{v}_{k}$, the $k$ th columns of $U$ and $V$, respectively, they satisfy

$$
\begin{gathered}
M \vec{v}_{k}=\epsilon_{k} \vec{u}_{k} \\
\vec{u}_{k}^{T} M=\epsilon_{k} \vec{v}_{k}^{T} .
\end{gathered}
$$

Let's find two unit-norm column vectors $\vec{u}$ and $\vec{v}$ and $\epsilon$ such that $M \vec{v}=\epsilon \vec{u}$ and $\vec{u}^{T} M=\epsilon \vec{v}^{T}$. First, we forget about the normalization and boundary conditions and focus on the secular equation. Setting the components of $\vec{u}$ and $\vec{v}$ as $u_{j}=$ $a x^{j}$ and $v_{j}=b x^{j}$, we have

$$
\begin{aligned}
M \vec{v} & =\frac{b}{a} \frac{\tau_{+}-\mu x+\tau_{-} x^{2}}{x} \vec{u}+\text { b.t., } \\
\vec{u}^{T} M & =\frac{a}{b} \frac{\tau_{-}-\mu x+\tau_{+} x^{2}}{x} \vec{v}^{T}+\text { b.t. }
\end{aligned}
$$

where b.t. stands for boundary terms. Hence, $u$ and $v$ satisfy the secular equation provided

$$
\frac{b}{a}=\sqrt{\frac{\tau_{-}-\mu x+\tau_{+} x^{2}}{\tau_{+}-\mu x+\tau_{-} x^{2}}}
$$

and

$$
\epsilon=\frac{1}{x} \sqrt{\left(\tau_{-}-\mu x+\tau_{+} x^{2}\right)\left(\tau_{+}-\mu x+\tau_{-} x^{2}\right)} .
$$

Because of the reflection symmetry $j \rightarrow N+1-j$, if $x$ is a solution of Eq. (A5) for some $\epsilon$, then $1 / x$ is also a solution. Assuming $\epsilon$ is known, the solutions are $x_{ \pm}$and $1 / x_{ \pm}$and satisfy

$$
\begin{aligned}
0 & =\epsilon^{2} x^{2}-\left(\tau_{-}-\mu x+\tau_{+} x^{2}\right)\left(\tau_{+}-\mu x+\tau_{-} x^{2}\right) \\
& \propto\left(x-x_{+}\right)\left(x-1 / x_{+}\right)\left(x-x_{-}\right)\left(x-1 / x_{-}\right),
\end{aligned}
$$

which by identification yields, writing $\rho_{ \pm}=x_{ \pm}+1 / x_{ \pm}$,

$$
\begin{aligned}
& x_{ \pm}=\frac{1}{2}\left(\rho_{ \pm}+\sqrt{\rho_{ \pm}^{2}-4}\right), \\
& \rho_{ \pm}=\frac{\mu t \pm \sqrt{\left(t^{2}-\Delta^{2}\right) \epsilon^{2}+\Delta^{2}\left(\mu^{2}-4 t^{2}+4 \Delta^{2}\right)}}{t^{2}-\Delta^{2}} .
\end{aligned}
$$

Taking into account the reflection symmetry, the general form of the components of $\vec{u}$ and $\vec{v}$ is thus

$$
\begin{aligned}
& u_{j}=a_{+} x_{+}^{j}+b_{+} x_{+}^{N+1-j}+a_{-} x_{-}^{j}+b_{-} x_{-}^{N+1-j}, \\
& v_{j}=a_{+} x_{+}^{N+1-j}+b_{+} x_{+}^{j}+a_{-} x_{-}^{N+1-j}+b_{-} x_{-}^{j},
\end{aligned}
$$

with the ratios $b_{+} / a_{+}$and $b_{-} / a_{-}$given by Eq. (A4) with $x=x_{+}$and $x=x_{-}$, respectively.

Furthermore, we have the boundary conditions

$$
\begin{aligned}
& a_{+}+b_{+} x_{+}^{N+1}+a_{-}+b_{-} x_{-}^{N+1}=0, \\
& a_{+} x_{+}^{N+1}+b_{+}+a_{-} x_{-}^{N+1}+b_{-}=0,
\end{aligned}
$$

which set the ratio $a_{-} / a_{+}$and give the quantization condition on the energies $\epsilon_{k}$. The last degree of freedom, say, $a_{+}$, is then set by normalizing $\vec{u}$ [from Eq. (A 8$),\|\vec{u}\|=\|\vec{v}\|$ ].

Note that for the special cases $t=\Delta$ and $\mu=0$, we have $\tilde{\gamma}_{1}^{\prime}=\gamma_{1}^{\prime}$ and $\tilde{\gamma}_{1}^{\prime \prime}=\gamma_{N}^{\prime \prime}$ with $\epsilon_{1}=0$. We have a similar result for $t=-\Delta$ and $\mu=0$. For these two cases, the general formalism described above does not apply since it yields $x_{ \pm}=0, \pm \infty$. 
[1] M. Z. Hasan and C. L. Kane, Rev. Mod. Phys. 82, 3045 (2010).

[2] X.-L. Qi and S.-C. Zhang, Rev. Mod. Phys. 83, 1057 (2011).

[3] F. D. M. Haldane, Phys. Lett. A 93, 464 (1983).

[4] T. Kennedy, J. Phys. Condens. Matter 2, 5737 (1990).

[5] F. Tedoldi, R. Santachiara, and M. Horvatić, Phys. Rev. Lett. 83, 412 (1999).

[6] A. Y. Kitaev, Phys. Usp. 44, 131 (2001).

[7] V. Mourik, K. Zuo, S. M. Frolov, S. R. Plissard, E. P. A. M. Bakkers, and L. P. Kouwenhoven, Science 336, 1003 (2012).

[8] S. Nadj-Perge, I. K. Drozdov, J. Li, H. Chen, S. Jeon, J. Seo, A. H. MacDonald, B. A. Bernevig, and A. Yazdani, Science 346, 602 (2014).

[9] S. Das Sarma, J. D. Sau, and T. D. Stanescu, Phys. Rev. B 86, 220506(R) (2012).

[10] R. Toskovic, R. van den Berg, A. Spinelli, I. S. Eliens, B. van den Toorn, B. Bryant, J.-S. Caux, and A. F. Otte, Nat. Phys. 12, 656 (2016).

[11] D. V. Dmitriev, V. Y. Krivnov, A. A. Ovchinnikov, and A. Langari, J. Exp. Theor. Phys. 95, 538 (2002).

[12] F. Mila, Nat. Phys. 12, 633 (2016).

[13] P. Pfeuty, Ann. Phys. (NY) 57, 79 (1970).

[14] This Hamiltonian is equivalent to an XY model in an in-plane magnetic field, but we chose to rotate the spins around the $x$ axis so that we recover the usual formulations of the TFI and LFI models as special cases.

[15] Reference [10] explains that the $\pm 3 / 2$ doublet of the spin-3/2 cobalt adatoms can be projected out by a Schrieffer-Wolff transformation due to the strong magnetic anisotropy. The resulting effective spin-1/2 model is that of Eq. (1) with $J_{x}=J_{z}$ and additional nearest-neighbor out-of-plane and next-nearestneighbor in-plane Ising couplings. These additional terms do not lead to qualitative changes because the model is still symmetric under a $\pi$ rotation of the spins around the $z$ axis, and since their coupling constants are small $\left(\sim 0.1 J_{x}\right)$, they have only a small quantitative effect in exact diagonalization results.
[16] S. Suzuki, J.-i. Inoue, and B. K. Chakrabarti, Quantum Ising Phases and Transitions in Transverse Ising Models, Lecture Notes in Physics Vol. 862 (Springer, Berlin, 2013).

[17] S. Jalal and B. Kumar, Phys. Rev. B 90, 184416 (2014).

[18] P. Ruján, Phys. Rev. B 24, 6620 (1981).

[19] F. Hassler and D. Schuricht, New J. Phys. 14, 125018 (2012).

[20] In the LFI model the lowest energy with a given magnetization $M=\sum_{i} S_{i}^{z}$ is $E_{0, M=0}=-J_{z}(N-1) / 4$ and $E_{0, M \neq 0}=E_{0,0}+$ $J_{z}(|M|-1 / 2)-M h$. Thus, for even $N$, the ground state has $M=0$ for $0<h<J_{z} / 2, M=1$ for $J_{z} / 2<h<J_{z}$, and $M=$ $N / 2$ for $h>J_{z}$, whereas for odd $N$ the ground state has $M=$ $1 / 2$ for $0<h<J_{z}$ and $M=N / 2$ for $h>J_{z}$.

[21] E. Lieb, T. Schultz, and D. Mattis, Ann. Phys. (NY) 16, 407 (1961).

[22] In the periodic chain used to get the mean-field parameters, there is a level crossing when $\mu=2 t$. To get good agreement with exact diagonalisation results and avoid a small discontinuity, we need to compute the expectation values in the state adiabatically connected to the ground state at $\mu<2 t$. Thus, for $\mu>2 t,\langle\cdot\rangle$ are not computed in the ground state but in the first excited state. Since all the level crossings arise for $\mu<2 t$, this has no influence on the following discussion.

[23] H. Katsura, D. Schuricht, and M. Takahashi, Phys. Rev. B 92, 115137 (2015).

[24] H.-C. Kao, Phys. Rev. B 90, 245435 (2014).

[25] S. S. Hegde and S. Vishveshwara, Phys. Rev. B 94, 115166 (2016).

[26] E. Barouch and B. M. McCoy, Phys. Rev. A 3, 786 (1971).

[27] D. Rainis, L. Trifunovic, J. Klinovaja, and D. Loss, Phys. Rev. B 87, 024515 (2013).

[28] S. M. Albrecht, A. P. Higginbotham, M. Madsen, F. Kuemmeth, T. S. Jespersen, J. Nygrd, P. Krogstrup, and C. M. Marcus, Nature (London) 531, 206 (2016).

[29] Y. Tserkovnyak and D. Loss, Phys. Rev. A 84, 032333 (2011). 